\title{
Success story of the SNAM Journal: fast progress into a well-established and reputable venue
}

\author{
Reda Alhajj
}

Published online: 23 February 2012

(C) Springer-Verlag 2012

Welcome to the first issue of the second volume of the Social Network Analysis and Mining (SNAM) journal. The SNAM journal project started 3 years ago to fill a gap and to satisfy a need. The target was to have a new journal that will be the leading venue in the field. Thus, SNAM is not yet another journal that will push hard to secure a share from the related articles available for publication. The response and reputation gained over the past short time, since the journal was announced in 2009 and after the first issue was published in January 2011, clearly justify that SNAM emerged to fill a gap and to satisfy a need. It has been well received and well recognized by researchers and practitioners. It is characterized by fast growing popularity and recognition at the international arena.

The SNAM journal is unique in its scope, coverage and mission. It brings together under one umbrella researchers and practitioners from a wide range of interrelated disciplines because their combined backgrounds and expertise are necessary to develop the theory and practice required to successfully tackle a vast range of emerging applications. Thus, it was decided to have the SNAM journal open to all type of article in the area of social network analysis and mining from technical to non-technical articles to cover the multidisciplinary nature of the research and hence help in meeting the expectations of the wide spectrum of readers, including both researchers and practitioners.

Researchers and practitioners are well aware of the fact that the SNAM journal complements ASONAM-the toptier and premier conference which has been identified by its technical sponsors and attendees as the flagship in the area

R. Alhajj (凶)

Department of Computer Science,

University of Calgary, Calgary, AB, Canada

e-mail: rsalhajj@gmail.com of social network analysis and mining. The two venues SNAM and ASONAM provided a great opportunity for a new community to emerge. The new community hosts researchers and practitioners from various disciplines; they work in harmony and agree upon combining the power of network construction and analysis with data mining and machine learning techniques to the benefit of a wide range of applications from social media modeling and analysis to biomedicine to finance and business analytics to web semantics and services to homeland security, among others.

The success of the SNAM journal and the ASONAM conference and their wide acceptance as premier and leading venues founded for and boosted the success of the Encyclopedia on Social Network Analysis and Mining (ESNAM) project which started 2 years ago and has recently entered the final stage. The first draft version of ESNAM will be completed by the end of 2012 and the first issue will be announced and published in Summer 2013. It is a solid and comprehensive piece of work that forms the umbrella and provides the necessary foundation for beginners, practitioners and researchers constituting the emerging community covering this promising and rapidly growing research area.

The SNAM journal is booming with rapid increase in the number and quality of submissions. Since the SNAM journal started 3 years ago there has been considerable interest from the international community of practitioners and researchers. It is well justified as a real international venue by hosting a strong board of associate editors who are elite researchers from North America, Europe, Asia and the Middle East. It is attracting submissions from leading researchers at the international arena. The ongoing progress is well in-lined with the mission because from the beginning the decision was to start big and to keep the target high. I decided to maintain high quality and to set high 
standards. I am happy to watch the SNAM journal progressing in short time into a well-established venue as one of the major and reputable outlets in the area of social network analysis and mining.

The SNAM journal is receiving more submissions than expected. Last year 50 was set as the anticipated target for the number of submissions. Springer officers and I had to work hard to increase the visibility of the journal and to attract more high quality submissions. By the end of December 2011 the number of submissions was close to 80 which is a record for a journal in its first year. Fortunately, Springer officers and I are working hard with the reviewers and associate editors to maintaining the short turnaround time for the reviewing process. Each article is reviewed by experts in the field who dedicate their time and effort to provide comprehensive and constructive reports. This seriousness increased the trust in the SNAM journal. On behalf of all parties involved in the SNAM journal, I heartedly thank all reviewers and associate editors who volunteered to help despite their busy schedules. It would not have been possible to achieve the success without the trust from the leading practitioners and researchers who submitted their articles, and the help and support from reviewers and associate editors who form the core of the emerging research community. Only $30 \%$ of the submissions were accepted during the first year. All accepted articles went through rounds of revision and re-evaluation before they entered into the production phase. This year the number of submissions is expected to hit a new record based on the number of submission received by the end of January 2012. The number of submissions in January 2012 has been doubled compared to January 2011. I hope the trend will continue and the SNAM journal will move forward to achieve new success.

In addition to the increase in the number of submissions, I am delighted to witness the SNAM journal gaining popularity over time. The number of visitors to the online version of the journal is increasing rapidly and the number of downloaded articles shows the increased visibility of and interest in the SNAM journal. I much appreciate the increased interest in the journal. Achieving the current success is only a step in the right direction. I am aware of the need to invest more time and effort to maintain the uprising trend.

Readers of this issue will enjoy the research achievements reported by researchers who are experts in social network analysis and mining. The articles in this issue show how the research in social networks has attracted considerable attention outside the basic disciplines where it originated, i.e., sociology and anthropology. I would like to thank all the contributing authors for the wonderful articles that will turn this issue into another valuable source for researchers and practitioners.
The first article "Profiling phishing activity based on hyperlinks extracted from phishing emails" by John Yearwood, Musa Mammadov and Dean Webb, describes a novel method for profiling phishing activity from an analysis of phishing emails. The authors formulate the profiling problem as a multi-label classification problem by considering hyperlink information. They reported testing results with quite high accuracy.

The second article "Iterative Multi-label Multi-relational Classification Algorithm for complex social networks" by Stéphane Peters, Yann Jacob, Ludovic Denoyer and Patrick Gallinari, tackles multi-label classification for data organized in a multi-relational graph. The authors propose a general algorithm for solving the inference and learning problems. They report interesting test results by covering two generic applications, image annotation and document classification, on different social datasets.

The third article "Positive influence dominating sets in power-law graphs" by Wei Zhang, Weili Wu, Feng Wang and Kuai Xu, focuses on the Positive Influence Dominating Set (PIDS) problem in power-law graphs. The authors prove that the greedy algorithm has a constant approximation ratio. They also provide some simulation results that demonstrate how the greedy algorithm can effectively select a small-scale PIDS set.

The fourth article "Social positions and simulation relations" by Joel Brynielsson, Lisa Kaati and Pontus Svenson, concentrates on simulation relation which creates a partial order on the set of actors in a network and shows how the order can be used to identify actors that have characteristic properties. The authors also show how the simulation relation can also be used to compute simulation equivalence which is a related but less restrictive equivalence relation than regular equivalence that is still computable in polynomial time.

The fifth article "Identifying the top-dogs of the blogosphere" by Justus Bross, Keven Richly, Matthias Kohnen and Christoph Meinel, addresses the development of a resilient weblog ranking metric within the global blogosphere. The authors analyze and compare the ranking criteria of several service providers.

The sixth article "Semantically interconnected social networks" by Alessandro Cucchiarelli, Fulvio D'Antonio and Paola Velardi, describes a methodology and a battery of tools to automatically extract from documents the relevant topics shared among community members and to analyze the evolution of the network also in terms of emergence and decay of collaboration themes. The authors conducted experiments on a scientific network funded by the European Community, the INTEROP network of excellence, and on the United Kingdom research community in medical image understanding and analysis. 
Last but not least, I would like to acknowledge the contributions of the hard workers behind the scene who have significant share to the achieved success. Various people from Springer deserve large credit for their help and support in all the issues related to the SNAM journal in general and in producing this first issue of the second volume in particular. I would like to thank Stephen Soehnlen for his continuing generous support in terms of time and effort required to keep the momentum. Stephen has always been positive. Unfortunately, Philipp Kammerer is no longer associated with the SNAM journal. I heartedly thank Philipp; he has been very patient in handling the requests and in following up with the timely production of the accepted articles. He has been recently replaced by Sudhakar Murugan who will be the main contact regarding the production of the accepted papers; I extend warm welcome to Sudhakar. Finally, I would like to thank Inaoba Singh for doing an amazing job taking care of the submissions system, handling all inquiries from the authors and alerting me whenever necessary.

Reda Alhajj

Editor-in-Chief

March 2012

Springer-Verlag Wien, NewYork 